Department of Anaesthesiology and Intensive Care Medicine | Head: Univ.-Prof. Dr. Bernd W. Böttiger

\title{
Hemodynamics of corpuls cpr in a Porcine Model of Cardiac Arrest
}

Tobias Neumann, Simon-Richard Finke, Pia Rademann*, Sebastian Lemke, Ben Höpfner, Maja Henninger, Daniel C. Schroeder, Thorsten Annecke

* Center for Experimental Medicine, University Hospital of Cologne

Purpose of the study

Whether mechanical chest compression devices can be used for Blood Pressure

cardiopulmonary resuscitation (CPR) is still a subject of debate.

Since data on chest compression quality unbiased by pharmacotherapy is rare we present current hemodynamic data on the fairly new corpuls cpr (GS Elektromedizinische Geraete G. Stemple $\mathrm{GmbH}$ ) from our animal model.

Materials and methods

Approved by the animal welfare authorities (LANUV NRW, reference 84-02.04.2017.A176), 10 domestic swine were sedated, ventilated and equipped with pressure transducers and a flow probe around the left carotid artery.

Following baseline (BL) measurement, ventricular fibrillation was induced by alternating current and ventilation was discontinued. After 5 minutes of untreated cardiac arrest mechanical CPR was After 5 minutes of untreated cardlac arrest, mechanical CPR was initiated (depth $6.0 \mathrm{~cm}$, continuous, 100 compressions per minute) and ventilation was resumed. Defibrillations were pharmacotherapy was applied.

Arterial blood pressure (SAP, DAP, MAP), endtidal carbon dioxide $\left(\right.$ etCO ${ }_{2}$ ) and mean carotid blood flow (CFmean) were averaged each 2 minute intervals (mean \pm SD).

Results

ROSC was achieved in 3 out of 10 animals. Hemodynamic data are presented in figures and tables.

Conclusions

Our data support another group's findings that corpuls cpr generates near to normal blood pressure and one third of baseline CFmean. [1] Therefore, we strongly encourage furthe clinical examination on corpuls cpr in comparison to manual CPR or other chest compression devices.

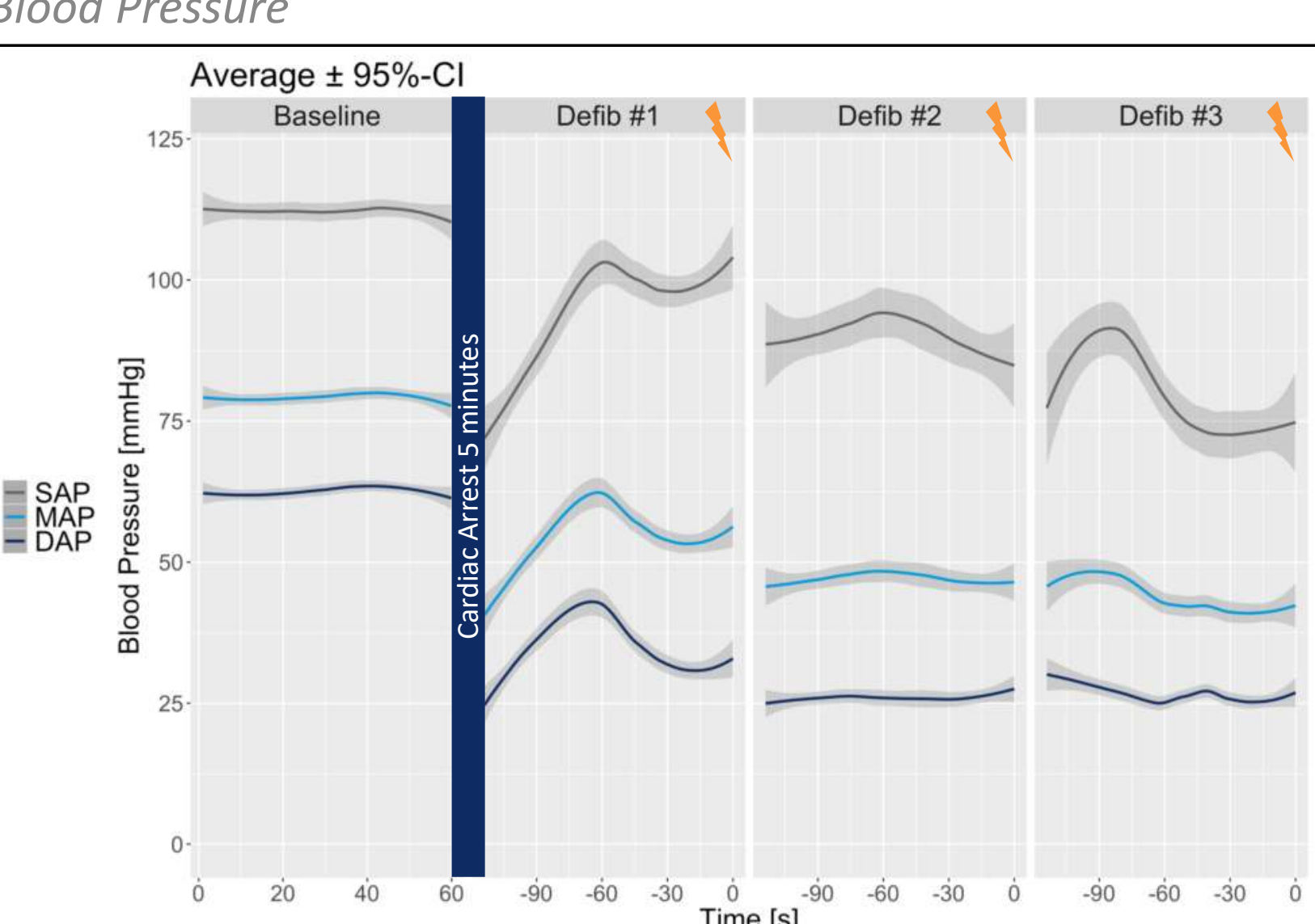

Carotid Blood Flow

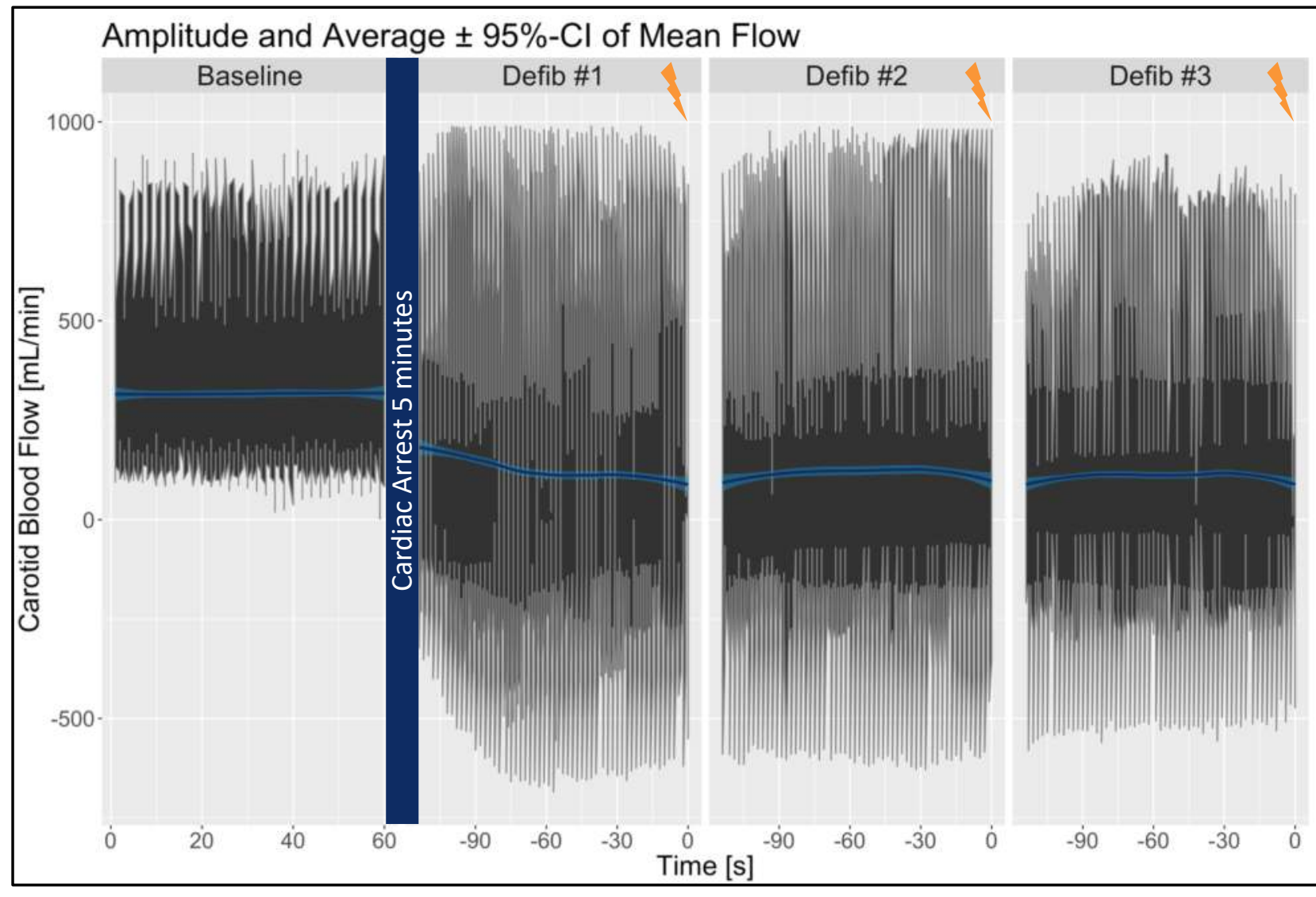

\begin{tabular}{|c|c|c|c|c|}
\hline & $\mathrm{BL}$ & $\begin{array}{c}\mathrm{CPR} \\
0-2 \mathrm{~min}\end{array}$ & $\begin{array}{c}\text { CPR } \\
2-4 \mathrm{~min}\end{array}$ & $\begin{array}{c}\text { CPR } \\
4-6 \mathrm{~min}\end{array}$ \\
\hline $\begin{array}{c}\text { SAP } \\
{[\mathrm{mmHg}]}\end{array}$ & $112 \pm 10$ & $93 \pm 28$ & $94 \pm 40$ & $78 \pm 27$ \\
\hline $\begin{array}{c}\text { MAP } \\
{[\mathrm{mmHg}]}\end{array}$ & $79 \pm 7$ & $53 \pm 17$ & $52 \pm 37$ & $43 \pm 12$ \\
\hline $\begin{array}{c}\text { DAP } \\
{[\mathrm{mmHg}]}\end{array}$ & $63 \pm 6$ & $33 \pm 15$ & $26 \pm 9$ & $26 \pm 8$ \\
\hline $\begin{array}{l}\text { etCO }_{2} \\
{[\mathrm{mmHg}]}\end{array}$ & $41 \pm 3$ & $27 \pm 5$ & $34 \pm 9$ & $41 \pm 9$ \\
\hline
\end{tabular}

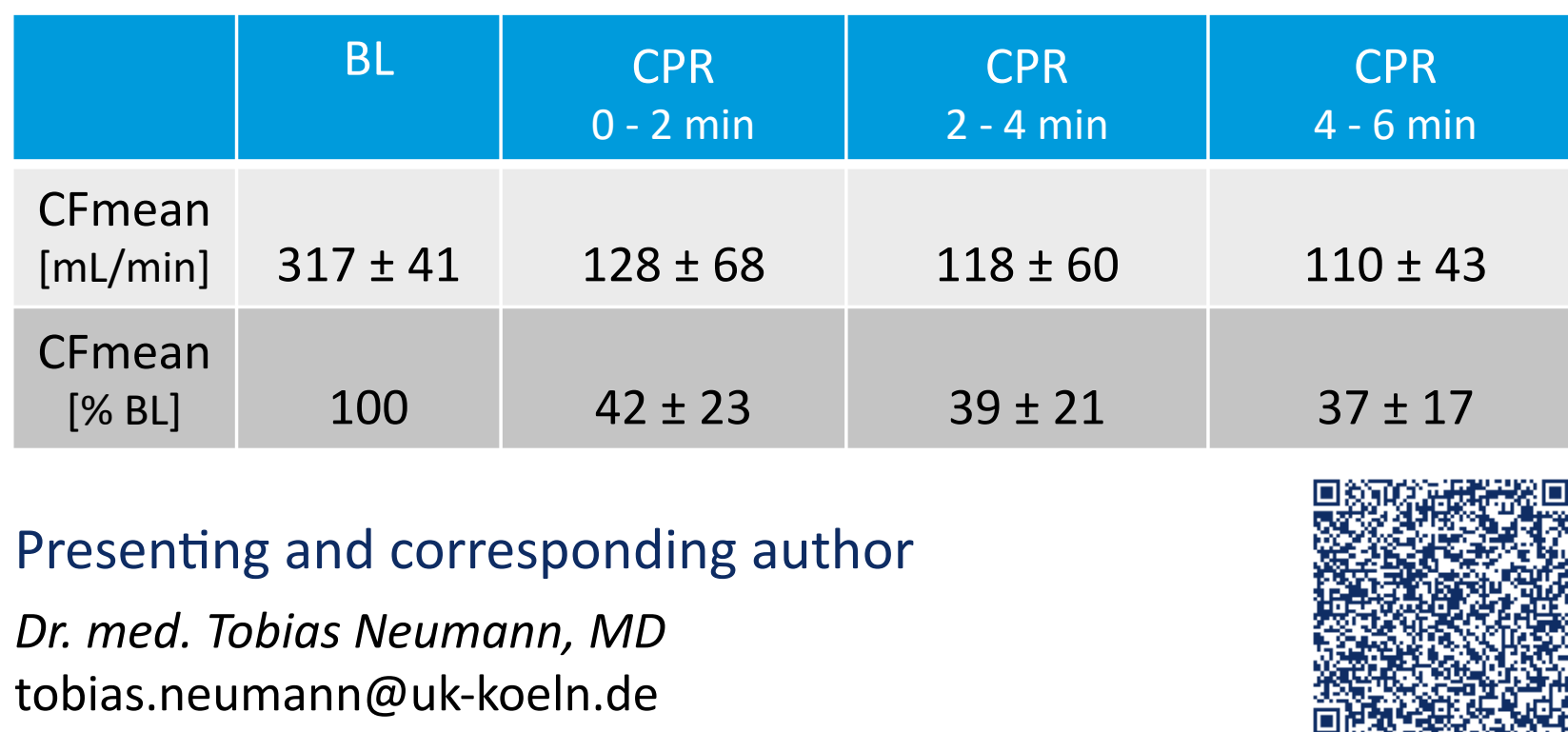

Reference

1) Eichhorn S, Mendoza A, Prinzing A, Stroh A, Xinghai L, Polski M, Heller M, Lahm H, Wolf E, Lange R, Krane M: Corpuls CPR Generates Higher Mean Arterial Pressure Than LUCAS Il in a Pig Model of Cardiac Arrest. BioMed Research International 2017:5470406. https://doi.org/10.1155/2017/5470406. 